\title{
Semiclassical chaos, the uncertainty principle, and quantum dissipation
}

\author{
Luca Bonci \\ Dipartimento di Fisica dell' Università di Pisa, Piazza Torricelli 2, 56100 Pisa, Italy \\ and Department of Physics, University of North Texas, Denton, Texas 76205 \\ Roberto Roncaglia and Bruce J. West \\ Department of Physics, University of North Texas, Denton, Texas 76205 \\ Paolo Grigolini \\ Dipartimento di Fisica dell' Università di Pisa, Piazza Torricelli 2, 56100 Pisa, Italy \\ and Department of Physics, University of North Texas, Denton, Texas 76205
}

(Received 4 December 1991)

\begin{abstract}
Using the Wigner method, it is shown that a classical-like equation of motion for a quasiprobability distribution $\rho_{W}$ can be built up, $\partial \rho_{W} / \partial t=\left(\hat{L}_{\mathrm{cl}}+\hat{L}_{\mathrm{QGD}}\right) \rho_{W}$, which is rigorously equivalent to the quantum von Neumann-Liouville equation. The operator $\hat{L}_{\mathrm{cl}}$ is equivalent to integrating classical trajectories, which are then averaged over an initial distribution, broadened so as to fulfill the requirements of the quantum uncertainty principle. It is shown that this operator produces semiclassical chaos and is responsible for quantum irreversibility and the fast growth of quantum uncertainty. Carrying out explicit calculations for a spin-boson Hamiltonian, the joint action of $\hat{L}_{\mathrm{cl}}$ and $\hat{L}_{\mathrm{QGD}}$ is illustrated. It is shown that the latter operator $\hat{L}_{\mathrm{QGD}}$ (where QGD stands for quantum generating diffusion), makes the $\frac{1}{2}$-spin system "remember" its quantum nature, and competes with the irreversibility induced by the former operator. Some ambiguous aspects of "irreversibility" and "growth of quantum fluctuations" as indicators of semiclassical chaos are discussed.
\end{abstract}

PACS number(s): 05.45.+b

\section{INTRODUCTION}

The importance of chaos in describing the dynamical behavior of classical systems has become increasingly important in the past decade [1]. Of course it has been known since Poincarés analysis of the three-body problem [2] that certain deterministic equations of motion have solutions that are so complex that they are indistinguishable from random [3]. This has resulted in the recent application of the concept of chaos to everything from weather prediction [4,5], to turbulence [6], to condensed-matter physics [7]. During this latter period investigators have also become concerned with how chaos in the classical domain is indicative of the evolution of systems in the quantum domain [8]. The traditional argument of quantizing the classical equations of motion in order to determine the evolution of the corresponding quantum equations breaks down when the classical equations are nonintegrable, as pointed out by Einstein in 1917 [9]. It has been argued by a number of investigators that the quantum manifestations of chaos are inhibited by physical mechanisms and therefore quantum phenomena should be less chaotic than their classical counterparts. In this we concur, but in so doing we introduce an argument more encompassing, to the authors' knowledge, than those presented previously. A number of such inhibitory mechanism have been identified and each argument describes the circumstances under which the classically observed effect of chaos is microscopically suppressed.
Note that, although quantum mechanics is more fundamental than classical mechanics in that the latter is obtained from the former in the domain where $\hbar$ is negligible compared with the typical action of the system, in the literature the form of the arguments might suggest that classical mechanics is the more fundamental. We believe this confusion to be semantic and not physical. The roots of classical chaos must be found in quantum mechanics and traced from the microcosm to the macrocosm, and one must not be misled by phrases such as "the quantum suppression of chaos." We are interested in those quantum systems that manifest special properties in the parameter regimes where their semiclassical descriptions are chaotic. These special properties need not involve chaos explicitly, but the question is from where does chaos emerge as $\hbar \rightarrow 0$.

There exist at least four theoretical explanations of what is called in the literature the "quantum suppression of classical chaos." The first [10] explanation relates this suppression to the "dynamical localization of diffusive excitation," which in analogy to Anderson's argument for a random potential relates classical chaos to wave-function localization [11]. In the example of a high-intensity electric field applied to a Rydberg atom [12] this localization process inhibits ionization [13]. In the second explanation it was argued that there exists a border in parameter space beyond which classical dynamics violates the quantum uncertainty principle [14]. The third explanation has to do with the number of quantum states coupled to the external field in the case of the Rydberg atom men- 
tioned above. This number drops precipitously with increasing frequency of the external field. Koch et al. [15] explain that one may use a severely truncated "quasiresonant" state basis [14] in which the effective density of states is much smaller than one would estimate at first, so that quantum effects become significant. Finally, it has been demonstrated by Mackey and Meiss [16] that classical chaos is suppressed when the phase-space area escaping through classical cantori (holes broken in Kolmogorov-Arnold-Moser surfaces) in each cycle of the electric field, is small compared to Planck's constant. Such cantori are opaque to quantum transport, but do not significantly impede classical transport until the driving field is raised to sufficiently high levels [15].

Herein we introduce a fifth explanation in that we use the Wigner pseudoprobability density [17] and a generalization of the attendant formalism to establish that the dynamics of a microscopic system can be explicitly separated into two pieces $[18,19]$. The Liouvillian describing the evolution of the Wigner density is $\hat{L}_{\mathrm{cl}}+\hat{L}_{\mathrm{QGD}}$; the first operator $\hat{L}_{\mathrm{cl}}$ gives the exact semiclassical description of the evolution of the system, i.e., the evolution in the $\hbar \rightarrow 0$ limit; the second operator $\hat{L}_{\text {QGD }}$ is the quantum generating diffusion (QGD) mechanism which has been recently shown to constrain the classical dynamics in such a way that the quantization prescriptions of quantum mechanics [20] are satisfied. If it were possible to ignore the action of the QGD mechanism, then we would make the following two predictions regarding the quantum effects of semiclassical chaos.

(i) The quantum fluctuations would undergo a massive growth, unexpected on the basis of standard quantum mechanics [21].

(ii) The time evolutions of the quantum expectation values would become irreversible and dissipative $[18,19]$.

Both property (i), which was recently pointed out by Fox [21], and property (ii), more recently discussed by Bonci et al. [18] and Roncaglia et al. [19], are immediately derived from the Wigner formalism. This is so because the adoption of the Wigner formalism supplemented by the neglect of the QGD mechanism is equivalent to the evaluation of the classical trajectories with a distribution of initial conditions. The initial distribution cannot be a $\delta$ function in the phase space variables in order to satisfy the quantum uncertainty principle. In the case where the classical approximation to the equation of motion yields chaotic trajectories this initial uncertainty is expected to grow exceptionally large, if not macroscopic as it did in the Fox paper [21]. On the other hand, since, according to the Wigner formalism, the time evolution of the expectation value of a quantum observable is formally obtained as an average over the corresponding classical trajectories, and these averages quickly lose their correlations when the trajectories are chaotic, the result is expected to be a relaxation. In other words, the time evolution of a quantum expectation value would be roughly equivalent to the correlation function of a random process $[18,19]$. We use numerical techniques to verify these theoretical expectations and to assess the role of the QGD mechanism on both quantum irreversibility and the growth of quantum fluctuations. We study these properties with the help of a spin-boson model and examine the dynamics of the oscillator (boson field) as well as that of the spin- $\frac{1}{2}$ system.

The outline of the paper is as follows. Section II is devoted to a brief review of the Wigner method. In Sec. III we extend the Wigner formalism to include spin and to initiate the study of the spin-boson Hamiltonian. The quantum irreversibility triggered by semiclassical chaos and its competition with the restraining role of the QGD mechanism is illustrated in Sec. IV for the spin- $\frac{1}{2}$ system. In Sec. $V$ we show that the sudden growth of quantum fluctuations is a manifestation of semiclassical chaos. Another, less marked manifestation, is the increased rate of the process of regression to equilibrium of the oscillator. It is also shown that the QGD mechanism markedly enhances the growth of quantum fluctuations. Section VI is devoted to concluding remarks.

\section{THE WIGNER DISTRIBUTION}

The Wigner distribution allows one to express quantum-mechanical averages in the same form one writes for classical averages. In this formalism every operator in Hilbert space, corresponding to a physical observable, is associated, via the Weyl transformation [22], to a function of suitable variables [17]. Consider a particle in one dimension with position operator $\hat{q}$ and momentum operator $\hat{p}$ such that the operator $\hat{A}(\hat{q}, \hat{p})$ has the associated function $A_{W}(q, p)$ :

$$
A_{W}(q, p)=\int_{-\infty}^{+\infty} d z \exp \left[i \frac{p}{\hbar} z\right]\left\langle q-\frac{z}{2}|\hat{A}| q+\frac{z}{2}\right\rangle \text {. }
$$

Equation (2.1) can be used to put the expectation value of the operator $\hat{A}$ into the form

$$
\langle\hat{A}(t)\rangle=\operatorname{Tr}[\hat{A} \hat{\rho}(t)]=\int d q d p A_{W}(q, p) \rho_{W}(q, p ; t),
$$

where $\rho_{W}(q, p ; t)$ is the Wigner pseudoprobability function associated with the density matrix $\hat{\rho}$,

$$
\rho_{W}(q, p ; t)=\frac{1}{\pi \hbar} \int_{-\infty}^{\infty} d z\langle q-z|\hat{\rho}| q+z\rangle \exp \left(2 i z \frac{p}{\hbar}\right) .
$$

Note the similarity between (2.2) and the classical definition of an average. For convenience, we shall refer to $\rho_{W}$ as the Wigner distribution or the Wigner density.

The most remarkable property of the Wigner distribution is its dynamical evolution, which also closely resembles the classical situation. Let us suppose that the quantum system is described by the following Hamiltonian:

$$
\hat{H}=\frac{\hat{p}^{2}}{2 m}+V(\hat{q})=-\frac{\hbar^{2}}{2 m} \frac{\partial^{2}}{\partial q^{2}}+V(\hat{q}) \text {. }
$$

The evolution of $\rho_{W}(q, p ; t)$ is then determined by [17] 


$$
\begin{aligned}
\frac{\partial}{\partial t} \rho_{W}(q, p ; t) & =\hat{L} \rho_{W}(q, p ; t) \\
& =-\left\{H, \rho_{W}\right\}+\sum_{n} \frac{1}{(2 n+1) !}\left(\frac{\hbar}{2 i}\right]^{2 n}\left[\frac{\partial^{2 n+1}}{\partial q^{2 n+1}} V(q)\right] \frac{\partial^{2 n+1}}{\partial p^{2 n+1}} \rho_{W}(q, p ; t),
\end{aligned}
$$

where the curly brackets denote the Poisson brackets, $H$ is the $c$-number Hamiltonian, $V(q)$ is the $c$-number potential, and $\hat{L}$ is the Liouville operator implicitly defined by (2.5). The main properties of the Wigner distribution are reviewed by Hillery et al. [17].

The most important features of the above Wigner formalism are summarized as follows.

(i) The concept of a quantum phase space is introduced in a natural way as the corresponding classical phase space. On the other hand, the state of the quantum system is not completely defined by its position in this phase space. In fact, due to the intrinsically statistical nature of quantum mechanics, even for a single quantum state, i.e., a pure state, the Wigner distribution cannot be a $\delta$ function. Thus a pure quantum state is not a point in this phase space, but is rather an ensemble of classical systems distributed according to the Wigner density. However, if this initial uncertainty undergoes a fast increase as an effect of semiclassical chaos, the resulting enhanced spreading must be interpreted as a quantum uncertainty of a single quantum system (enhanced by truly quantummechanical fluctuations).

(ii) Equation (2.5) introduces another kind of dynamical evolution for quantum systems. Following the standard treatment of statistical mechanics we define the dynamical operator for the function $A_{W}(q, p ; t)$ as

$$
\frac{\partial A_{W}(q, p ; t)}{\partial t}=\hat{\Gamma} A_{W}(q, p ; t) .
$$

The operator $\hat{\Gamma}$ must satisfy the equation

$$
\begin{aligned}
\langle\hat{A}(t)\rangle & =\int d q \int d p A_{W}\left[\exp (\hat{L} t) \rho_{W}\right] \\
& =\int d q \int d p\left[\exp (\hat{\Gamma} t) A_{W}\right] \rho_{W},
\end{aligned}
$$

where we have used (2.2). Note that in the limit $\hbar \rightarrow 0$ the operator $\hat{L}$ recovers the exact classical result, i.e., it becomes the Poisson bracket with the Hamiltonian [cf. (2.5)] which is just the classical Liouvillian.

Following the above procedure we have that (2.1) and (2.6) give the phase-space equivalent of the Heisenberg representation of quantum mechanics. The formalism is our starting point in the analysis of the behavior of the solutions to the Schrödinger (or Heisenberg) equation and we find it to be singularly useful for study of the quantum system whose classical limit is nonintegrable and chaotic.

Note that the average over the initial conditions must satisfy the inequality

$$
U \equiv \Delta q \Delta p \geq \hbar / 2,
$$

where $\Delta q$ and $\Delta p$ are the root-mean-square quantities

$$
\begin{aligned}
& \Delta q(t)^{2} \equiv\left\langle q(t)^{2}\right\rangle-\langle q(t)\rangle^{2}, \\
& \Delta p(t)^{2} \equiv\left\langle p(t)^{2}\right\rangle-\langle p(t)\rangle^{2} .
\end{aligned}
$$

Equation (2.8) is one expression of the quantum uncertainty principle (QUP), which within the Wigner formalism results in a broadened classical distribution at all times, including the initial one. Note that in the present illustrative case, the classical approximation cannot result in chaos, due to the one-dimensional character of the system under study, i.e., we have a single $q$ and a single $p$. However, one can easily imagine the generalization of this approach to the $n$-dimensional case, where classical chaos is admitted. In the chaotic case the initial broadening of the Wigner density forced by the QUP is expected to increase at a rate determined by the Liapunov coefficients and independently of the QGD mechanism [21]. However, as we show with numerical methods in Sec. V, the growth of $U$ is limited by the finite size of the phase space available to a deterministic system, and the quantum fluctuations becoming macroscopic according to the prediction of Fox [21] are possible only if this phase space region is macroscopic. On the other hand, an expectation value is the average over many trajectories, which quickly lose correlations with the initial conditions and among themselves when the trajectories are chaotic. Thus the time evolution of an expectation value is expected to manifest the character of irreversibility.

Actually, these remarks do not take into account the fact that, due to the presence of the QGD mechanism quantum mechanics is not simply obtained by averaging over classical trajectories. Due to the presence of a derivative of order greater than unity in (2.6), unlike the classical case, the operator $\hat{\Gamma}$ is not deterministic, i.e., $[\exp (\widehat{\Gamma} t)(A B)] \neq[\exp (\hat{\Gamma} t) A][\exp (\hat{\Gamma} t) B]$.

In the phase-space representation the evolution of quantum systems is determined by the interplay of the above two contributions, the classical Liouvillian and the QGD mechanism: the first leads to an evolution of the quantum system in a way completely equivalent to the corresponding classical one, with initially broadened distributions mimicking the uncertainty principle. When the classical or semiclassical approximation is affected by chaos, it is expected to produce irreversibility and a rapid increase in the strength of the quantum fluctuations; the second operator modifies this evolution to take the constraints posed by quantization rules into account.

\section{SPIN-BOSON HAMILTONIAN}

Let us examine the application of the Wigner formalism to a quantum-mechanical system of sufficient complexity that its semiclassical equations of motion have chaotic solutions. We investigate one of the simplest of such systems, this being the spin-boson Hamiltonian:

$$
\hat{H}=-\frac{1}{2} \omega_{0} \hat{\sigma}_{z}+\frac{g}{\sqrt{2 \Omega}} \hat{\sigma}_{x}\left(\hat{b}+\hat{b}^{\dagger}\right)+\Omega \hat{b}^{\dagger} \hat{b},
$$


where $\hat{b}\left(\hat{b}^{\dagger}\right)$ is the annihilation (creation) operator with commutation relation $\left[\hat{b}, \hat{b}^{\dagger}\right]=1$. The physical system modeled by (3.1) is that of a spin- $\frac{1}{2}$ dipole interacting with a magnetic field directed along the $z$ axis and modeled by an oscillator of frequency $\Omega$ with space coordinate $\hat{q}=\left(\hat{b}+\hat{b}^{\dagger}\right) / \sqrt{2 \Omega}$ and momentum $\hat{p}=i\left(\hat{b}^{\dagger}-\hat{b}\right) \sqrt{\Omega / 2}$. Note that $\hat{\sigma}_{x}$ and $\hat{\sigma}_{z}$ are spin matrices. Herein we study the dynamics of the spin-boson system with the initial condition

$$
\hat{\sigma}_{z}| \pm\rangle= \pm| \pm\rangle
$$

and the boson field is in a state characterized by the average number of photons $\langle n\rangle$.

A system of this kind has recently been studied by Graham and Höhnerbach [23] and Fox and Eidson [24]. They show that a semiclassical approximation of the system with Hamiltonian (3.1) exhibits chaotic dynamics for sufficiently strong coupling $g$ when the system is in resonance. Similar results have been previously found by Belobrov, Zaslavskii, and Tartakovskii [25] and Milonni, Ackerhalt, and Galbraith [26].

Further insight into the behavior of this system can be obtained using the Wigner formalism. Let us extend the arguments used earlier so as to be applicable to the spinboson problem. For this system the Wigner density reads

$$
\begin{aligned}
\rho_{W}(\mathbf{x}, q, p ; t)=\int & d \mathbf{k} \frac{\exp (-i \mathbf{k} \cdot \mathbf{x})}{(2 \pi)^{3}} \\
& \times \int d \alpha d \tau \frac{\exp -i(q \alpha+p \tau)}{(2 \pi)^{2}} F(\mathbf{k}, \alpha, \tau ; t),
\end{aligned}
$$

where $F(\mathbf{k}, \alpha, \tau ; t)$ is the quantum characteristic function defined by

$$
\begin{gathered}
F(\mathbf{k}, a, \tau ; t)=\operatorname{Tr}\left\{\exp \left[i\left(k_{1} \widehat{\sigma}_{x}+k_{2} \hat{\sigma}_{y}+k_{3} \widehat{\sigma}_{z}\right)\right]\right. \\
\times \exp [i(\alpha \hat{q}+\tau \hat{p})] \hat{\rho}(t)\},
\end{gathered}
$$

where $\hat{\rho}(t)$ is the density operator for the complete quantum system. Note that (3.3) and (3.4) extend the usual treatment of the Wigner distribution to include spin.

The variables $\mathbf{x} \equiv\left(x_{1}, x_{2}, x_{3}\right), p$ and $q$ are the phasespace variables associated, via a generalized Weyl rule of the form (2.1), to the spin-boson operators: $\hat{\sigma}_{j} \rightarrow x_{j}$, $\hat{q} \rightarrow q, \hat{p} \rightarrow p$. The quantum average values of these operators on the statistical system described by the density matrix $\hat{\rho}(t)$ are given by

$$
\begin{aligned}
& \left\langle\widehat{\sigma}_{j}(t)\right\rangle=\int d \mathbf{x} d q d p x_{j} \rho_{W}(\mathbf{x}, q, p ; t), \\
& \langle\widehat{q}(t)\rangle=\int d \mathbf{x} d q d p q \rho_{W}(\mathbf{x}, q, p ; t) .
\end{aligned}
$$

The corresponding equation of evolution for the Wigner distribution (3.3) is given by

$$
\frac{\partial}{\partial t} \rho_{W}(\mathbf{x}, q, p ; t)=\left(\hat{L}_{\mathrm{cl}}+\hat{L}_{\mathrm{QGD}}\right) \rho_{W}(\mathbf{x}, q, p ; t),
$$

where after some substantial algebra we obtain for the spin-boson system $[18,19]$

$$
\begin{aligned}
\hat{L}_{\mathrm{cl}} \equiv & \omega_{0}\left[x_{1} \frac{\partial}{\partial x_{2}}-x_{2} \frac{\partial}{\partial x_{1}}\right]+2 g q\left[x_{3} \frac{\partial}{\partial x_{2}}-x_{2} \frac{\partial}{\partial x_{3}}\right] \\
& +\Omega^{2} q \frac{\partial}{\partial p}-p \frac{\partial}{\partial q}+g x_{1} \frac{\partial}{\partial p}
\end{aligned}
$$

and

$\hat{L}_{\mathrm{QGD}} \equiv g\left[\frac{\partial}{\partial x_{1}}-\frac{\partial}{\partial x_{1}} x_{1}^{2}-\frac{\partial}{\partial x_{2}} x_{1} x_{2}-\frac{\partial}{\partial x_{3}} x_{1} x_{3}\right]$.

The operator $\hat{L}_{\mathrm{cl}}$ is identical to the Liouvillian of a classical dipole interacting with a classical oscillator, i.e., this term alone corresponds to the semiclassical set of equations discussed by various authors [23-26]. We refer to the calculations based on the study of the single trajectory solutions of the nonlinear dynamical equations as the semiclassical predictions. In this regard we point out a significant feature of the analysis that was apparently overlooked in previous investigations having to do with chaotic trajectories [23-26]. These earlier studies focused on individual trajectories and gave them physical meaning. However, from (3.5) and (3.6) we see that even when $\hat{L}_{\text {QGD }}$ is neglected, it is only the ensemble that has physical significance not the individual trajectories [18].

The term $\hat{L}_{\mathrm{QGD}}$ in (3.9) has a number of significant implications. It has a diffusionlike structure, but the state dependence of the "diffusion coefficient" results in its not being positive definite. It has recently been shown by Roncaglia et al. [20] that if the oscillator is coupled to a heat bath so as to transmit to the spin- $\frac{1}{2}$ dipole standard thermal fluctuations, then this term results in the average value of the $z$ component of the dipole changing from a Langevin (classical) function to the hyperbolic tangent (quantum). In other words, this term was coined quantization generating diffusion by these authors [20], precisely because it ensures that the dipole retains its quantum nature. The operator $\hat{L}_{\mathrm{QGD}}$ acts as an antiduffusional mechanism, it competes against thermal fluctuations and constrains the dipole, which otherwise would freely diffuse over all possible orientations, to vacillate between two possible orientations. In Sec. V, however, we show that the influence of the QGD mechanism on the motion of the oscillator is qualitatively different from that exerted on the spin- $\frac{1}{2}$ system. This is in part expected, since there are no intuitive reasons why the state quantization of an oscillator should impede diffusion. We shall see via numerical calculation that both dissipation and the rate of the growth of quantum fluctuations are enhanced by the QGD mechanism. The quantitative account of this effect is left as a subject for a future investigation.

In Ref. [19] it is shown in detail that if the QGD mechanism is neglected, then the solution of (3.7) is equivalent to determining the trajectories described by the system of equations

$$
\begin{aligned}
& \dot{x}_{1}(t)=\omega_{0} x_{2}(t), \\
& \dot{x}_{2}(t)=-\omega_{0} x_{1}(t)-2 g q(t) x_{3}(t), \\
& \dot{x}_{3}(t)=2 g q(t) x_{2}(t), \\
& \dot{q}(t)=p(t), \\
& \dot{p}(t)=-\Omega^{2} q(t)-g x_{1}(t) .
\end{aligned}
$$


It must be pointed out that the numerical solution of (3.10) supplemented by averaging over the initial Wigner density is equivalent to solving (3.7) with the QGD mechanism neglected.

\section{NUMERICAL RESULTS ON THE MOTION OF THE SPIN- $\frac{1}{2}$ SYSTEM}

We here study the time evolution of $\left\langle\widehat{\sigma}_{z}(t)\right\rangle$ starting from an initial condition described by

$$
\hat{\rho}(0)=|+\rangle\langle+| \hat{\rho}_{B}(0),
$$

which means that the spin- $\frac{1}{2}$ dipole is initially polarized along the $z$ axis and the boson field is initially in a state characterized by the density matrix $\hat{\rho}_{B}(0)$. We carry out our investigation with the following two distinct initial conditions for the boson field.

(i) The boson field is in a coherent state with photon number defined by $\langle n\rangle=N$. This is a state of minimum uncertainty.

(ii) The boson field is in the eigenstate $|n\rangle$ of the boson Hamiltonian $\Omega \hat{b}^{\dagger} \hat{b}$ with $n=N$.

The calculation of the time evolution of $\left\langle\hat{\sigma}_{z}(t)\right\rangle$ is carried out in two different ways. The first approach consists in determining the single trajectories of (3.10) and then averaging these orbits over the initial Wigner density corresponding to the state (4.1). As already said, this is equivalent to solving (3.7) without the QGD mechanism. The second approach yields the exact result, including the effect of the QGD mechanism, and is obtained by solving the von Neumann-Liouville equation numerically, corresponding to the Hamiltonian of (3.1). To demonstrate the irreversibility properties associated with classical chaos we also make an exact calculation to determine the entropy for the spin- $\frac{1}{2}$ dipole system

$$
S(t)=-\operatorname{Tr}_{\text {spin }}\left[\hat{\rho}_{s}(t) \ln \hat{\rho}_{s}(t)\right],
$$

where

$$
\hat{\rho}_{s}(t)=\operatorname{Tr}_{\text {boson }}[\hat{\rho}(t)] \text {. }
$$

Section $\mathrm{V}$ will be devoted to the study of the motion of the oscillator subsystem with the initial condition (i). Both conditions refer to a pure state. Thus, according to a theorem by Araki and Lieb [27], the entropy of the boson system must coincide with the entropy of the spin- $\frac{1}{2}$ system. Thus the numerical results concerning the entropy (4.2) can also be regarded as being an indication of the irreversibility affecting the oscillator motion. We also evaluate the entropy of the oscillator subsystem as a check on the accuracy of our numerical calculations, and we find it to virtually coincide with the entropy of the spin- $\frac{1}{2}$ system, thereby employing a very high numerical accuracy.

We see from Figs. 1(a) and 2(a) that upon changing from the condition $\omega_{0}<<\Omega$ to the resonance condition $\omega_{0}=\Omega$, the time evolution of $\left\langle\hat{\sigma}_{z}(t)\right\rangle$ changes its character from the collapse-revival behavior into that of a distinctly relaxationlike process. It has been pointed out that the collapse-revival behavior is fitted very well by a linear theory [28] which emphasizes the reversible char- acter of the process when $\omega_{0}<<\Omega$. In this case the Larmor frequency $\omega_{0}$ is so weak as to produce a negligible reaction field and a virtually deterministic semiclassical limit. When the resonance condition $\omega_{0}=\Omega$ is reached the Liapunov coefficients become large and positive [19] and correspondingly, the calculations ignoring the influence of the QGD mechanism result in a type of irreversible behavior. This is so because the mean value $\left\langle\hat{\sigma}_{z}(t)\right\rangle$ is a consequence of averaging the spin matrix over many trajectories, which, due to the semiclassical chaos quickly lose their correlations. Whereas on the scale of the off-resonance case of Fig. 1(a), two theories (the exact calculation and the analytical theory of Ref. [28]) coincide and are in remarkably good agreement with the result of the Wigner method without the QGD term, in the resonant case of Fig. 2(a) the Wigner method without the QGD mechanism results in a more pronounced relaxation behavior than the exact numerical treatment (which coincides with the result of the Wigner method when the QGD mechanism is retained). This is
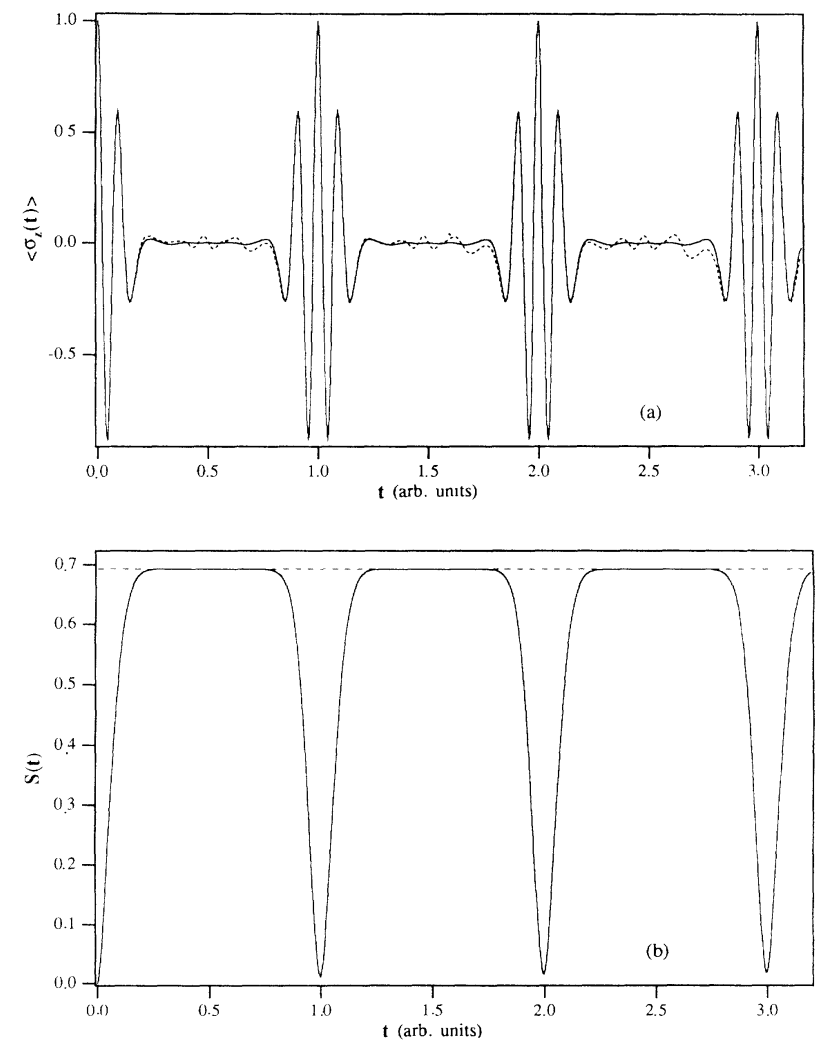

FIG. 1. Off-resonance dynamics of the spin- $\frac{1}{2}$ system. In all curves $g=20, \omega_{0}=10^{-2}, \Omega=2 \pi$, and the initial condition (i) of Sec. IV, namely the oscillator in the coherent state, with $\langle n\rangle=10$. (a) Time evolution of $\left\langle\sigma_{z}(t)\right\rangle$. The solid curve is the analytic prediction of Ref. [28]. In the scale of this figure this theoretical prediction coincides with the "exact" numerical result. The dashed curve is obtained using the Wigner equation without the QGD mechanism. (b) Time evolution of the entropy $S(t)$ of spin- $\frac{1}{2}$ system. The solid curve is "exact" numerical result. The dashed line is the theoretical maximum value of the entropy. 
consistent with the conclusion drawn in Ref. [20] that the QGD mechanism counterbalances the action of diffusion. It must be stressed that these remarks apply to the orientational diffusion of the spin- $\frac{1}{2}$ dipole. Quantization rules imply that only two directions of this dipole are admitted, and free diffusion away from these two admitted orientations produced by $\hat{L}_{\mathrm{cl}}$ must be inhibited in some way.

The calculation of the entropy confirms the generation of irreversibility triggered by semiclassical chaos [see Figs. 1(b) and 2(b)]. It must be pointed out that this is distinct from the behavior of the entropy of the same system, studied under the rotating-wave approximation (RWA) by Phoenix and Knight [29]. In their case [29] the entropy is shown to exhibit a sort of overall increase, though not as marked as in the case of Fig. 2. However, this latter increase has to do with the fact that a coherent state can mimic a bath with an infinite number of degrees of freedom [18]. The collapses occur because of the many different frequencies contributing to the time evolution of the mean values of interest, one for each eigenstate $|n\rangle$ of the unperturbed Hamiltonian of the oscillator $(n=0,1, \ldots)$ necessary to build a coherent state. The re-
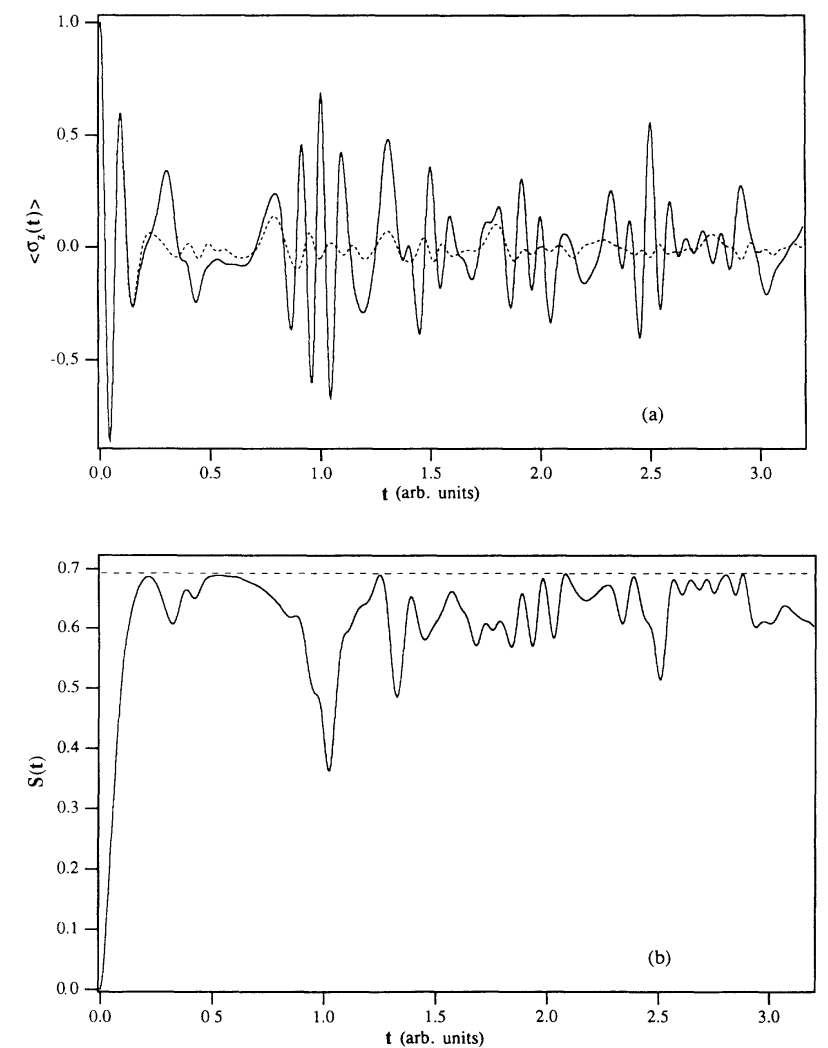

FIG. 2. Resonance dynamics of the spin- $\frac{1}{2}$ system. In all curves $g=20, \omega_{0}=\Omega=2 \pi$, and the initial condition (i) of Sec. IV, namely the oscillator in the coherent state, with $\langle n\rangle=10$. (a) Time evolution of $\left\langle\sigma_{z}(t)\right\rangle$. The solid curve is the "exact" numerical result. The dashed curve is obtained using the Wigner equation without the QGD mechanism. (b) Time evolution of the entropy $S(t)$ of spin- $\frac{1}{2}$ system. The solid curve is "exact" numerical result. The dashed line is the theoretical maximum value of the entropy. vivals are not perfect because the frequency involved go as $n^{1 / 2}$, not as an integer multiple of $n$ [29]. As remarked in Ref. [18], to make it clear that we are dealing with another kind of irreversibility, it is convenient to reduce the boson field to a single phonon as given by condition (ii). In this case the RWA leads to reversible behavior, whereas our exact calculations, with no RWA approximation, show that the entropy of the system exhibits a behavior more and more distinctly irreversible upon increase of $g$ (see Fig. 3).

Note that the behavior illustrated by the time evolution of the entropy $S$ is the result of an exact calculation, thereby including the effect of the QGD mechanism. We see that the QGD mechanism does not completely destroy dissipation. The ghosts of reversible behavior are manifest by dips at integer multiple of $\Omega$, which have, however, a decreasing intensity as time increases, thereby implying a global irreversibility for sufficiently large coupling coefficient $g$.

\section{NUMERICAL RESULTS ON THE MOTION OF THE OSCILLATOR}

In this section we illustrate the results of calculations on the dynamics of the oscillator utilizing the initial condition (i) of Sec. IV. We also monitor the time evolution of the corresponding uncertainty $U$ [cf. Eq. (2.8)]. An observation of the same kind, on the oscillator rather than on the spin- $\frac{1}{2}$ dipole system, was recently made by Müller et al. [30]. These authors used the Husimi [31] rather than the Wigner density to build up the quantum Poincare maps for the oscillator. As correctly stated by these authors, the Wigner density, due to violent oscillations between positive and negative values, cannot be used for this purpose. However, we want to stress that their study of the quantum Poincare map did not lead them to realize that the growth of the quantum uncertainty due to semiclassical chaos persists until the maximum possible value of $U$ admitted by the finite size of the available phase space is obtained.

It must be pointed out that since the region of phase space available to the oscillator has a finite size, the growth of $U$ must be limited from above. Thus we expect that the growth of $U(t)$ is characterized by the attainment of an asymptotic value for $t$ going to infinity. If our arguments on the growth of quantum fluctuations as a result of chaos are correct, we expect that the phenomenon of the growth of fluctuations to be negligible when the RWA is made since this approximation is known to be incompatible with chaos [23-26].

These theoretical expectations are checked using both the solution without the QGD mechanism and the exact numerical solution of the quantum-mechanical Liouville equation (i.e., the solution of the Wigner equation of motion, also including the QGD mechanism).

Figure 4 shows that our theoretical expectation on the semiclassical chaos as a source of fast growth of quantum fluctuations is correct. In this figure we show the time evolution of $U(t)$, as it results from an "exact" numerical 

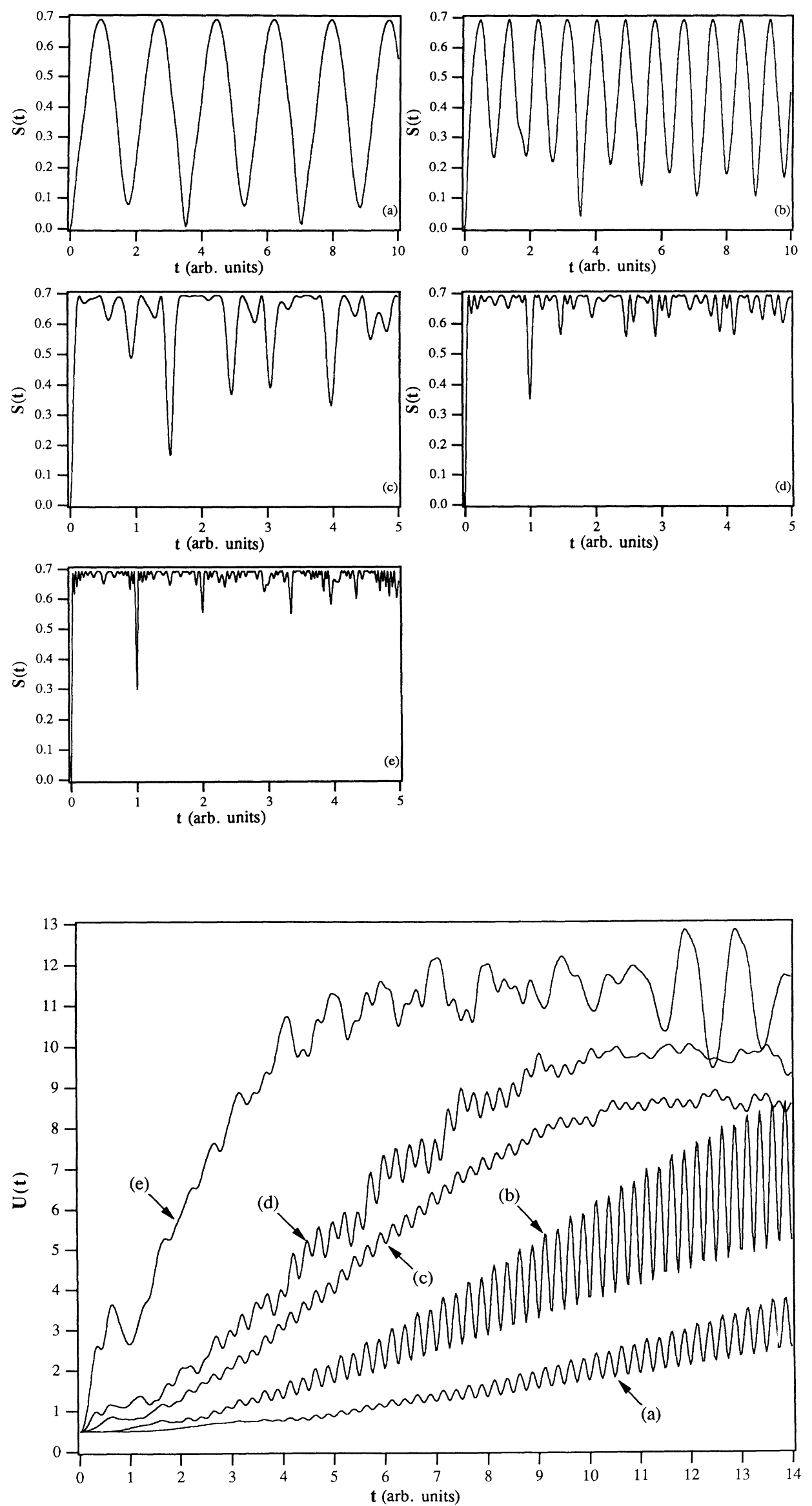

FIG. 3. The time evolution of the entropy of the spin- $\frac{1}{2}$ system subsequent the initial condition (ii) (the boson field in the eigenstate $|n\rangle$ with $n=N)$. The resonance condition $\omega_{0}=\Omega=2 \pi$ is considered. We use the following parameters: (a) $N=10$, $g=1$; (b) $g=2$; (c) $g=5$; (d) $g=10$; (e) $g=20$.

FIG. 4. The quantum uncertainty $U(t)$ as a function of time, calculated with no approximation. The starting point is condition (i) of Sec. IV, namely the oscillator in the coherent state, with $\langle n\rangle=10$. The resonance condition $\omega_{0}=\Omega=2 \pi$ is considered. We use the following parameters: (a) $g=1$; (b) $g=2$; (c) $g=5$; (d) $g=10$; (e) $g=20$. 
calculation, upon increasing the interaction strength $g$. The Liapunov coefficients are not a monotonic function of $g$ [19]. However, for the five values of $g$ chosen, larger $g$ 's involve larger Liapunov coefficients. Curve $(e)$ correspond to a fully chaotic regime for the equation of motion. We thus see that classical chaos is consistent with the quantum-mechanical uncertainty quickly reaching a fixed asymptotic value. This signals that quantum fluctuations grow so fast as to quickly invade the whole region of the available phase space.

Figure 5 illustrates the relaxationlike nature of the time evolution of $\langle q(t)\rangle$. This character is more distinct when the QGD mechanism is included. The comparison with the RWA condition points out an element of ambiguity of "irreversibility" as a proper indicator of semiclassical chaos. As mentioned earlier in this paper (see the remarks in Sec. IV on the entropy of Ref. [29] concerning the RWA Hamiltonian), the initial condition with the oscillator in the coherent state supplemented by the RWA can generate some degree of irreversibility. This is due to the fact that the dynamics of the system de-
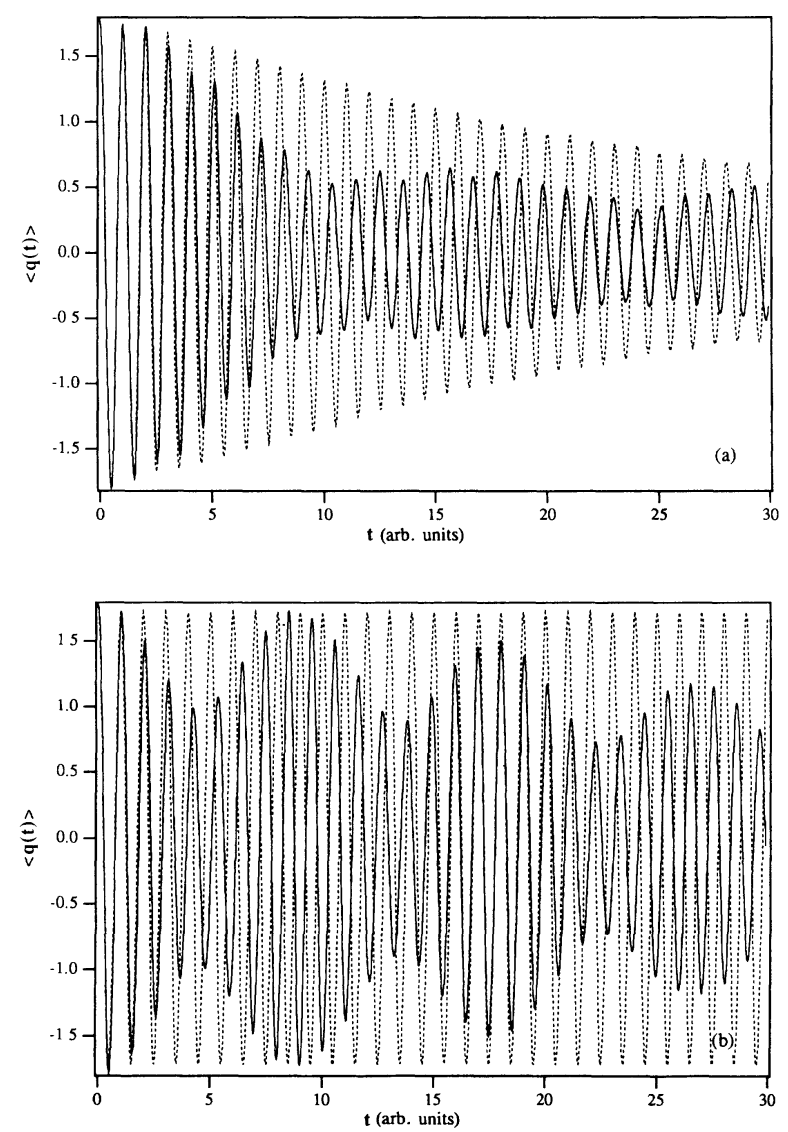

FIG. 5. Time evolution of $\langle q(t)\rangle$. In all curves $g=10$, $\omega_{0}=\Omega=2 \pi$, and the starting point is condition (i) of Sec. IV, namely the oscillator in the coherent state, with $\langle n\rangle=10$. The full line is the result of the "exact" numerical calculation. The dashed line denotes the result of averaging on the semiclassical trajectories over the Wigner density corresponding to the above condition (i). (a) refers to the complete Hamiltonian, with no RWA and (b) to the RWA. pends on an infinite number of eigenstates of the oscillator with incommensurate frequencies [29], and this is reflected in a overall increase of the entropy of the oscillator system. The results illustrated in Fig. 5(b) on the irreversibility of $\langle q(t)\rangle$ under the RWA reflects this property. We see indeed that, whereas the averaging of the RWA trajectories over the condition (i) of Sec. IV results in a nondissipative motion, the full quantum-mechanical RWA calculation exhibits some elements of dissipation, albeit less marked than in case without RWA. We believe that semiclassical chaos is manifest in a relaxation behavior of the oscillator, but this is mixed in this case with the irreversibility stemming from the conventional source of a heat bath played by the coherent state.

Figure 6 shows that the QGD mechanism increases the rate of growth of quantum fluctuations. A quite surprising result illustrated in Fig. 6 is that of the quantum RWA. The initial increase of $U$ of this case is the most violent. We see that it grows very quickly, much faster than any other process; it reaches a maximum, then it undergoes a fast decrease until it reaches a first minimum. For simplicity of description, we are not taking into account the ultrafast oscillations of this curve. The process is repeated over and over again with minima of ever increasing value. The envelope of the maxima of this curve would correspond to a process of growth for the uncertainty $U(t)$ comparable to that triggered by semiclassical chaos. However, if we focus our attention on the envelope of the minima, we see that this turns out to be, as must be, an increase faster than the semiclassical RWA case, but slower than the predictions of both quantummechanical calculations without RWA.

The reason why the actual increase of quantum uncertainty is indicated by the envelope of the minima [curve (e) of Fig. 6] is given by an interesting quantummechanical property recently discovered by Eiselt and Risken [31]. The Eiselt-Risken effect is a quantum phenomenon associated with the well-known quantum effect of collapses and revivals [29]. The collapse process is shown [32] to correspond to a splitting of the quantum cloud into two distinct clouds. These two clouds quickly move far apart, thereby provoking a fast increase of $U$. The minima are easily explained by the fact that periodically, and precisely in the correspondence of the revivals, the two distinct clouds merge again into a single cloud. In conclusion, in this case $U$ is not a reliable indicator of the quantum uncertainty, since the splitting of the cloud into two clouds results in a larger $U$, whereas the total volume of the quantum cloud (the sum of the volumes of the two single clouds) does not change appreciably compared to the volume of the cloud before splitting. If we replace the function $U(t)$ with the envelope of its minima [curve $(e)$ ], then we see that our theoretical expectations [that for a case with semiclassical chaos the rate of increase $U(t)$ evaluated with the RWA is slower than without RWA] are fulfilled.

\section{CONCLUDING REMARKS}

Adopting the Wigner formalism we have established two important quantum manifestations of semiclassical 


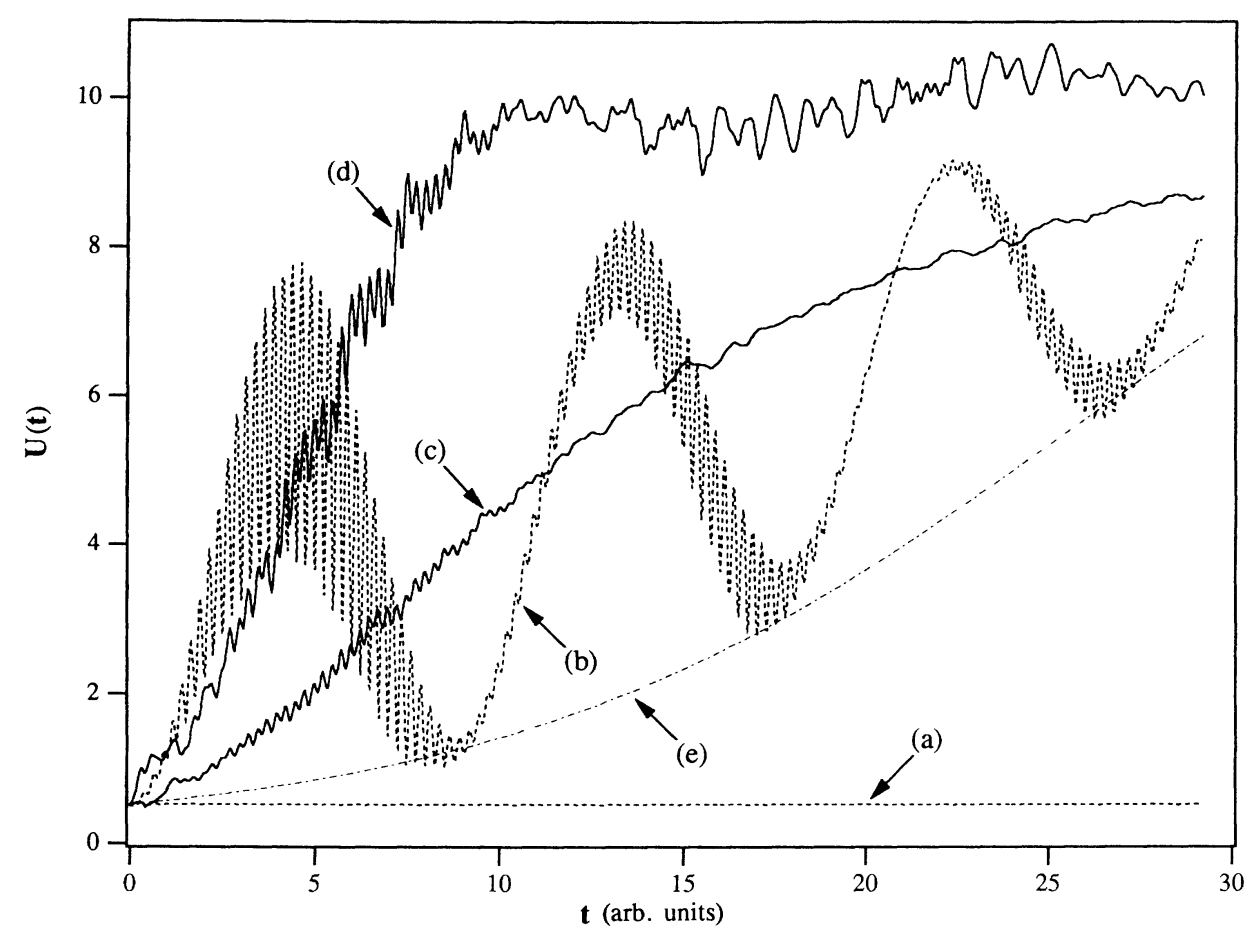

FIG. 6. The quantum uncertainty $U(t)$ as a function of time. In all curves $g=10, \omega_{0}=\Omega=2 \pi$, and the starting point is condition (i) of Sec. IV, namely the oscillator in the coherent state, with $\langle n\rangle=10$. Curves $(a)$ and $(b)$ refer to the RWA approximation. ( $a$ ) is the result of a calculation without the QGD mechanism and $(b)$ is the result of a fully quantum mechanical RWA treatment. Curve $(e)$ is a guide for the eye and indicates the effective increase of quantum uncertainty, if we disregard the Eiselt-Risken effect [32]. Curves $(c)$ and $(d)$ refer to a calculation made with no RWA approximation. Curve $(c)$ is the result of a calculation neglecting the QGD mechanism, whereas $(d)$ is the result of a calculation with no approximation.

chaos: these are irreversibility and unexpected growth of quantum uncertainty. Surprisingly enough these quantum manifestations of semiclassical chaos are accounted for by using classical arguments, namely, the classical contribution to the Wigner equation of motion, $\hat{L}_{\mathrm{cl}}$ of (3.8), and the sensitive dependence on initial conditions of classical chaos. Quantum mechanics, though, is subtlety involved, since the QUP sets a constraint on the initial Wigner density so that it cannot be a mere Dirac $\delta$ function. Thus adopting classical arguments and the QUP, one would be led to conclude with Fox [21] that "to properly describe classical-mechanical chaos, one must do quantum mechanics." In conclusion, on the latter property we substantially agree with the point of view of Fox [21]. However, according to the theoretical analysis of Fox, based on the adoption of arguments similar to the expansion of the master equation in terms of the macroscopic volume $(1 / \hbar$ is the macroscopic parameter of his analysis), the growth of quantum uncertainty is proved to depend only on what we call $\hat{L}_{\mathrm{cl}}$. In the spin-boson model here under study (it must be noticed that the Fox analysis was applied in Ref. [21] to the multidimensional version of the nonlinear oscillator studied for illustrative purposes in Sec. IV, and not to the spin-boson model of the present paper) we see, on the contrary, that also the QGD mechanism contributes to the growth of quantum uncertainty and makes it grow significantly faster than it does with this mechanism absent. In a future publication we will give theoretical arguments to account for the speeding up role of the QGD mechanism on the growth of the quantum uncertainty of the oscillator. In the case where the semiclassical equations of motion have chaotic solutions, the quantum fluctuations that in Ref. [21] are expected to become macroscopic, within the spin-boson model of this paper are shown to quickly fill the phase space which would be explored by the single semiclassical and chaotic trajectory. This means an extended cloud of quantum uncertainty the size of which is the available phase space. The consequences of this property might be quite remarkable, since distinct regions of the same quantum cloud should bear precise phase relations (with the capability of producing interference effects of a single particle with itself), and this appears to conflict with the fact that within the semiclassical approximation the same phase-space region is the domain of randomness.

The former consequence of semiclassical chaos, quantum irreversibility, is intimately connected to the latter, but surprisingly it has been overlooked by the investigations carried out so far in the field of quantum chaos. Quantum irreversibility as a manifestation of semiclassical chaos is distinct from the irreversibility generated by the interaction of the system with a bath with many degrees of freedom. The assumption that the radiation field, the oscillator of our model, is in a coherent state, produces irreversibility features [29] which are reminiscent of those observed in this paper (see Fig. 2). It must be noticed indeed that Phoenix and Knight study the RWA approximation of the spin-boson model and thus in 
their case irreversibility is not in any way related to semiclassical chaos, since the RWA suppresses semiclassical chaos [23-26]. However, in Fig. 3 we show that semiclassical chaos and quantum irreversibility have the same source even with a starting condition which would result in reversible behavior within the RWA approximation. This is clear evidence of the existence of the kind of irreversibility provoked by chaos rather than by the action of an infinite number of degrees of freedom in a heat bath.

If we focus our attention on the oscillator dynamics, we find that if the Eiselt-Risken effect [31] is properly taken into account, then the growth of quantum fluctuations turns out to be a more reliable indicator of semiclassical chaos than is irreversibility. This is so again because irreversibility is already manifest within the RWA [29]. Even in this case the effect of the QGD mechanism is to make the relaxation rate larger. It must be remarked that the QGD mechanism seems to exert on the oscillator an effect different from that exerted on the spin- $\frac{1}{2}$ system. The regression to equilibrium of the mean value $\left\langle\hat{\sigma}_{z}(t)\right\rangle$ is characterized indeed by more marked revivals if the QGD mechanism is taken into account. This can be intuitively explained by noticing that the QGD mechanism makes the system "remember" its quantum nature, thereby limiting the diffusion processes [20]. This certainly applies to the spin- $\frac{1}{2}$ dipole, which has only two possible orientations and cannot freely diffuse as a result of chaos (chaos-induced diffusion). No constraint of this kind is set on the oscillator. The oscillator moves within an energy potential, which is left harmonic by its interaction with the spin- $\frac{1}{2}$ system. The only remarkable effect of this interaction is that the restoring center of the oscillations moves according to the chaotic dynamics of the spin- $\frac{1}{2}$ system. This does not set any constraint on the oscillator diffusion. In conclusion, the numerical results of this paper show that the QGD mechanism makes the dynamics of the spin- $\frac{1}{2}$ system "less dissipative" and the dynamics of the oscillator "more dissipative." Roughly speaking, we can say that the final result is that both subsystems are "equally dissipative," since, according to a theorem by Araki and Lieb [27] on the quantum entropy of two subsystems, the choice of a pure state as the initial condition of the whole system makes the entropy of the oscillator coincide with the entropy of the spin- $\frac{1}{2}$ system. It must be remarked that, as a check on our numerical calculations, we evaluated both the entropy of the spin- $\frac{1}{2}$ system and that of the oscillator separately, and found that they virtually coincide with one another. The theoretical reasons why the QGD mechanism makes the oscillator "more dissipative" are planned to be explored in a future publication.

We want to stress that both quantum "irreversibility" and the growth of the quantum uncertainty are not completely unambiguous indicators of the underlying semiclassical chaos. We have already remarked that the entropy of the RWA system with the oscillator in a coherent state exhibits elements of irreversibility [29].
The irreversibility manifested by semiclassical chaos on the dynamics of the spin- $\frac{1}{2}$ system is, however, much more marked than the irreversibility of the corresponding RWA system. The problem posed by the growth of $U$ within the RWA seems to be a much more serious problem. $U(t)$ exhibit larger oscillations, and the envelope of the resulting maxima seems to be the fastest process for the attainment of the saturation regime. However, this is so for reasons which are well understood, i.e., the EiseltRisken effect [32], and does not weaken the importance of this interesting effect of semiclassical chaos. Semiclassical chaos results in the fastest increase of the total volume of the quantum cloud, in spite of the fact that $U(t)$ exhibits the fastest process of initial increase within the quantum RWA treatment.

As a final remark, we again stress that we do not wish to give the impression that we consider classical mechanics more fundamental than quantum mechanics, since the anomalous increase of quantum-mechanical uncertainty and the irreversible nature of the time evolution of the quantum mean value $\left\langle\widehat{\sigma}_{z}(t)\right\rangle$ is derived from the properties of the semiclassical approximation. This misinterpretation might arise because, according to our derivation, if the QGD term of (3.9) can be neglected, quantum mechanics is obtained by making averages over the chaotic semiclassical trajectories. This procedure leads immediately to the prediction of irreversibility and the anomalous growth of quantum uncertainty. It would be much more difficult to make these predictions from within the linear confines of quantum mechanics, even though the latter is more fundamental than the former. If we adopt the correct perspective of regarding classical mechanics as derived from quantum mechanics, then the formalism developed herein shows why the semiclassical approximation leads to a chaotic behavior, not exhibited by the linear equation of quantum physics. The semiclassical approximation makes it possible to introduce the concept of semiclassical trajectories and the semiclassical equation of motions are nonlinear, whereas the corresponding classical Liouvillian, as well as the complete quantum Liouvillian, is linear. As a consequence in quantum mechanics, chaos is destroyed in precisely the same way as it would be in classical physics, if attention were focused on the time evolution of the probability distribution rather than on single trajectories. We further remark that if a classical system is chaotic, then the only way to recover predictability, is to give a statistical meaning to the classical density function. If we assume this perspective, then the striking discrepancy between semiclassical (or classical) chaos and quantum regularity is drastically reduced, the only difference left being that produced by the QGD term.

\section{ACKNOWLEDGMENT}

We thank the Texas Higher Education Coordinating Board for partial support of this research (Texas Advanced Research Program, Project No. 003594-038). 
[1] G. M. Zaslavsky, R. Z. Sagdeed, D. A. Usikov, and A. A. Chernikov, Weak Chaos and Quasi-Regular Patterns (Cambridge University Press, Cambridge, England, 1991).

[2] H. Poincaré, Acta Math. 13, 1 (1890).

[3] J. Moser, Stable and Random Motions in Dynamical Systems (Princeton University Press, Princeton, 1973), Vol. 77.

[4] E. N. Lorenz, J. Atmos. Sci. 20, 130 (1963).

[5] Predictability of Fluid Motions (Scripps Institution of Oceanography of the University of California, San Diego), Proceedings of a Symposium on Fluid Dynamics, AIP Conf. Proc. 106, edited by Greg Holloway and Bruce J. West (AIP, New York, 1983).

[6] D. Ruelle and F. Takens, Commun. Math. Phys. 20, 167 (1971); 23, 343 (1971).

[7] Stochastic Behavior in Classical and Quantum Hamiltonian Systems, edited by G. Casati and J. Ford, Lecture Notes in Physics Vol. 93 (Springer, Berlin, 1979).

[8] M. Tabor, Chaos and Integrability in Nonlinear Dynamics (Wiley, New York, 1989).

[9] A. Einstein, Verh. Dtsch. Phys. Ges. 19, 82 (1917).

[10] B. V. Chirikov, F. M. Izrailev, and D. L. Shepelyanskii, Theor. Math. Phys. 43, 417 (1980).

[11] S. Fishman, D. R. Grempel, and R. E. Prange, Phys. Rev. Lett. 49, 509 (1982).

[12] R. V. Jensen, Phys. Rev. 304, 386 (1984).

[13] G. Casati, B. V. Chirikov, and D. L. Shepelyanskii, Phys. Rev. Lett. 53, 2525 (1984).

[14] J. G. Leopold and D. Richard, Phys. Rev. A 38, 2660 (1988).

[15] P. M. Koch, L. Moorman, B. E. Sauer, and E. J. Galvez, in Classical Dynamics in Atomic and Molecular Processes
(World Scientific, Singapore, 1989).

[16] R. S. Mackey and J. D. Meiss, Phys. Rev. A 37, 4702 (1988).

[17] M. Hillery, R. F. O'Connell, M. O. Scully, and E. P. Wigner, Phys. Rep. 106, 122 (1984).

[18] L. Bonci, R. Roncaglia, B. J. West, and P. Grigolini, Phys. Rev. Lett. 67, 2593 (1991).

[19] R. Roncaglia, L. Bonci, P. Grigolini, and B. J. West, J. Stat. Phys. (to be published).

[20] R. Roncaglia, R. Mannella, D. Vitali, and P. Grigolini (unpublished).

[21] R. F. Fox, Phys. Rev. 41, 2969 (1990).

[22] H. Weyl, Z. Phys. 46, 1 (1927).

[23] R. Graham and M. Höhnerbach, Z. Phys.-Condens. Matter 57, 233 (1984).

[24] R. F. Fox and J. Eidson, Phys. Rev. 34, 482 (1986).

[25] P. I. Belobrov, G. M. Zaslavskii, and G. Kh. Tartakovskii, Zh. Eksp. Teor. Fiz. 71, 1799 (1976) [Sov. Phys. -JETP 44, 945 (1976)].

[26] P. W. Milonni, J. R. Ackerhalt, and H. W. Galbraith, Phys. Rev. Lett. 50, 966 (1983); 51, 1108(E) (1983).

[27] H. Araki and E. Lieb, Commun. Math. Phys. 18, 160 (1970).

[28] L. Bonci, P. Grigolini, and D. Vitali, Phys. Rev. A 42, 4452 (1990).

[29] S. J. D. Phoenix and P. L. Knight, Ann. Phys. (N.Y.) 186, 381 (1988); Phys. Rev. Lett. 66, 2833 (1991).

[30] L. Müller, J. Stolze, H. Leschke, and P. Nagel, Phys. Rev. A 44, 1022 (1991).

[31] K. Husimi, Proc. Phys. Math. Soc. Jpn. 22, 246 (1940).

[32] J. Eiselt and H. Risken, Phys. Rev. A 43, 346 (1991). 